\title{
A Framework for Processing Partially Free Word Order*
}

\author{
Hans Uszkoreit \\ Artificial Intelligence Center \\ SRI International \\ 333 Ravenswood Avenue \\ Menlo Park, CA 94025
}

\begin{abstract}
The partially free word order in German belongs to the class of phenomena in natural language that require a close interaction between syntax and pragmatics. Several competing principles, which are based on syntactic and on discourse information. determine the linear order of noun phrases. A solution to probiems of this sort is a prerequisite for high-quality language generation. The linguistic framework of Generalized Phrise Structure Grammar oflers tools for dealing with -word order variation. Some slight modifieations to the framework allow for an analysis of the German data that incorporates just the right degree of interaction between syntactic and pragmatic components and that can account for conflicting ordering statements.
\end{abstract}

\section{Introduction}

The relatively free order of major pbrasal constituents in German belongs to the class of natural-language phenomena that require a closer interaction of syntax and pragmatics than is usually accounted for in formal linguistic frameworks. Computational linguists who pay attention to both syntax and pragmatics will find that analyses of such phenomena can provide maluable dat a for the design of systems that integrate these linguistic components.

German represents a good test case because the rcle of pragmatics in governing word order is much greater than in English $w$ bile the role statax plays is greater than in some of the so-called free-word-order languages like Warlpiri. The German dat a are well attested and thoroughly discussed in the descriptive literature. The fact that English and German are closely related mates it easier to assess these data and to draw parallels.

The simple analysis presented here for dealing with free word order in German syntax is based on the linguistic framework of Generalized Phrase Structure Grammar (GPSG), especially on its Immediate Dominance/Linear Precedence formalism (ID/LP), and complements an earlier treatment of

"This research was supported by the National Science Foundation Grant IST-81035.50. The views and conclusions expressed in this paper are those if the authir and should not be interpreted as representative of the views If the Nistiunal Science Foundation or the United States government. I have benefited from discussions with and comments from Barbara Grosa, Fernando Pereira, Jane Robinson, and Stuart Shieber.
German word order. ${ }^{1}$ The framework is slightly modified to accommodate the relevant class of word order regularities.

The syntactic framework presented in this paper is not bound to any particular theory of discourse processing; it enables syntax to interact with whatever formal model of pragmatics one might want to implement. A brief discussion of the framework's implications for computational implementation centers upon the problem of the status of metagrammatical devices.

\section{The Problem}

German word order is essentially tixed: however. there is some freedom in the ordering of major phrasal categories like NPs and adverbial phrases - for example, in the linear order of subject (SLBJ), direct object (DOBJ), and indirect object (IOBJ) with respect to one another. All six permutations of these three constituents are possible for sentences like (1a). Two are given as (1b) and (lc).

(1a) Dann hatte der Doktor dem Mann die Pille gegeben. Then bad the doctor the man the pill given

(1b) Dann batte der Doktor die Pille dem Mann gegeben. Then had the doctor the pill the man given

(IC) Dann batte die Pille der Doktor dem Mann gegeben. Then had the pill the doctor the man given

All permutations bave the same truth conditional meaning, which can be paraphrased in Eaglish as: Then the doctor gave the man the pill

There are several basic principles that influence the ordering of the thrce major NPs:

- The unmarked order is SLIBJ-IOBJ-DOBJ

- Comment (or locus) follows non-comments

- Personal pronouns precede ocher NPs

- Light constituents precede beavy constituents.

\footnotetext{
'The best overvicw of the current GPSG framework can be found in Gazdar and Pullum (1882). For 3 description of the ID/LP format refer to Gazdar and Puilum (1981) and Klein (1983), for the ID/LP trestment of German t. ('szkureil (19823, 1882b) and Nerbonne (1982).
} 
The order in (1a) is based on the unmarked order, (1b) would be appropriate in a discourse situation that makes the man the focus of the sentence, and (1c) is an acceptable sentence if both doctor and man are focussed upon. I use focus bere in the sense of comment, the part of the sentence that contains new important information. (1c) could be uttered as an answer to someone who inquires about both the giver and recipient of the pill (for example, with the question: Who gave whom the pill?). The most complete description of the ordering principles, especially of the conflict between the unmarked order and the topic-comment relation, can be found in Lenerz (1977).

\section{Implications for Processing Models}

Syntactic as well as pragmatic information is needed to determine the right word order; the unmarked-order principle is obviously a syntactic statement, whereas the topiccomment order principle requires access to discourse information.2 Sometimes different ordering principles make contradictory predictions. Example (1b) violates the unmarked-order principle; (1a) is acceptable even if dem Mann (the man) is the foc us of the sentence. ${ }^{3}$

The interaction of ordering variability and pragmatics can be found in many languages and not only in so-called free-wordorder languages. Consider the following two English sentences:

(2a) I will talk to him after lunch about the offer.

(2b) I will talk to him about the offer after lunch.

Most semantic frameworks would assign the same truthconditional meaning to (2a) and (2b), but there are discourse situations in wich one is more appropriate than the other. (2a) can answer a question about the topic of a planned afternoon meeting, but is much less likely to occur after an order to mention the offer as soon as possible.

Formal linguistic theories have traditionally assumed the existence of ratber independent components for syntax, semanties, and pragmatics. ${ }^{5}$ Linguistics not only could afford this idealization but has probably temporarily benefited from it. However, if the idealization is carried over to the computational implementation of a framework. it can have adverse effects on the efficiency of the resulting system.

\footnotetext{
2The heaviness principle requires access to phonological information in ad. dition, but 3 diseussion of this dependence is beyond the scope of this paper

${ }^{3}$ Sentences that differ only in their discourse role assignments, e.g., do not focus on the same constituent(s), usually exhibit different sentential stress patterns.

The claim is not that these sentences are not interchangeable in the mentir.ned discriurse situations under any circumstances. In English. marked inination can usually overwrite default discourse role assignments associated with the irder of the consticuents.

${ }^{5}$ Several more recent theories can account for the interaction among some of the components. Montague Grammar (Montague, 1974) and its successors (incl. GPSG) link semantic and syntzctic rules. Work on presuppositions (Kartiunen and Peters. 1979), discourse representations (Kamp, 1980) and Siluation Semantics (Barwise and Perry, 1981) narrows the gap between stemantics and pragmatics.
}

If we assume that a language generation system should be able to generate all grammatical word orders and if we further assume that every generated order should be appropriate to the given discourse situation, then a truly nonintegrated system, i.e., a system whose semantic, syntactic, and pragmatic components apply in sequence, has to be inefficient. The syntax will first generate all possibilities, after which the pragmatic cumponent will have to select the appropriate variant. To do so, this componcnt will also need access to syntactic information.

In an integrated model, much unnecessary work can be saved if the syntax refrains from using rules that introduce pragmatically inappropriate orders. A truly integrated model can discard improper parses very early during parsing, thereby considerably reducing the amount of syntactic processing.

The question of integrating grammatical components is a linguistic problem. Any reasonable solution for an integration of syntax and pragmatics has to depend on linguistic fadings about the interaction of syntactic and pragmatic phenomena. An integrated implementation of any theory that does not account for this interaction will either augmeat the theory or neglect the linguistic facts.

By supporting integrated implementations, the framework and analysis to be proposed below fulfill an important condition for efficient treatment of partially free word order.

\section{The Framework and Syntartic Analysis}

\subsection{The Framework of CPSG in ID/LP Format}

The theory of GPSG is based on the assumption that nat ural languages can be generated by context-free phrase structure (C'F-PS) grammars. As we know, such a grammar is bound to exbibit a bigh degree of redundancy and. consequently, is not the right formalism for encoding many of the linguistic generalizations a framework for natural language is expected to express. However. the presumption is that it is possible to give a condensed inductive definition of the CF.PS grammar, w bich contains various componeats for encoding the linguistic regularitics and which can be interpreted as a metagrammar, i.e., a grammar for generating the actual CF-PS grammar.

A GPSG can be defined as a two-level grammar containing a metagrammar and an object grammar. The object grammar combines (CF-PS) syntax and model-theoretic semantics. its rules are ordered triples $\langle n, r, t\rangle$ where $n$ is an integer (the rule number), $r$ is a CF-PS rule, and $t$ is the translation of the rule, it denotation represented in some version of intensional logic. The translation $t$ is actually an operation that maps the translation of the children nodes iato the translation of the parent. The nonterminals of $r$ are complex symbols, subsets of a finite set of syntactic features or - as in the latest version of the theory (Giazliar and Pullum, 1982) - feature trees of finite size. The rules of the object grammar are interpreted as tree-admissability conditions.

The metagrammar consists of four different kinds of rules that are used by three major components to generate the object 
grammar in a stepwise fashion. Figure (3) illustrates the basic structure of a GPSG metagrammar.

(3)

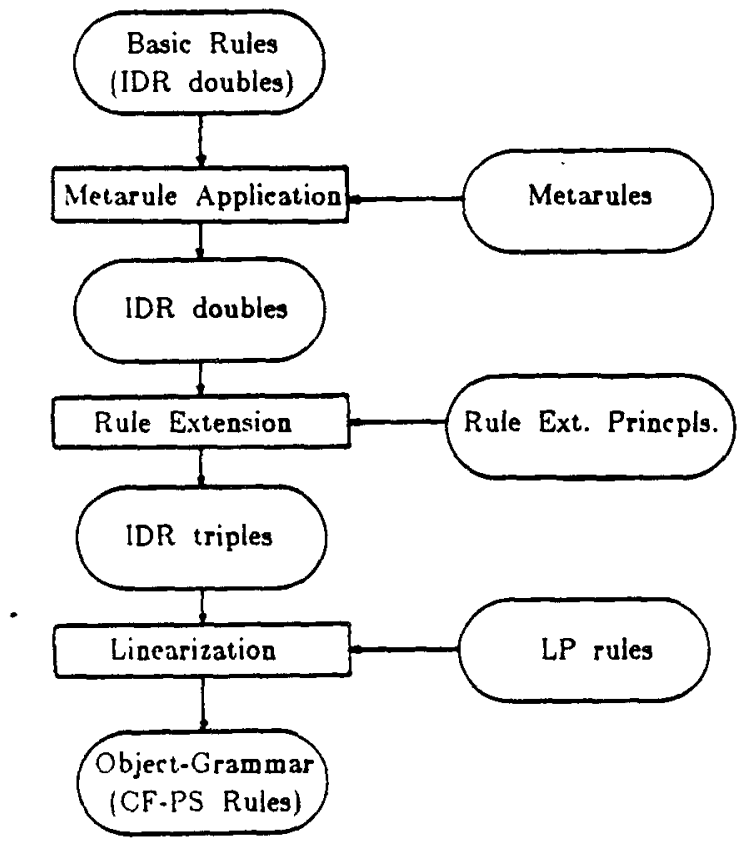

First. there is a set of basic rules. Basic rules are immediate dominance rule (IDR) doubles, ordered pairs $\langle n, i\rangle$, where $n$ is the rule number and $i$ is an IDR.

IDRs closely resemble CF.PS rules, but, whereas the CF. PS rule $y \rightarrow \delta_{1} \delta_{2} \ldots \delta_{n}$ contains information about both immediate dominance and linear precedence in the subtree to be sccepted. the corresponding IDR $\gamma \rightarrow \delta_{1}, \delta_{2}, \ldots, \delta_{n}$ encodes only information about immediate dominance. The order of the right-hand-side symbols, which are separated in IDRs by commas, has no significance.

Ifetarule Application, maps IDR doubles to otber IDR doubles. For this purpose, metarules, which are the second kind of rules are applied to basic rules and then to the output of metarule applications to generate more IDR doubles. Metarules are relations between sets of IDRs and are written as $A \Rightarrow B$, where $A$ and $B$ are rule templates. The metarule can be read as: If there is an IDR double of kind $A$, then there is also an IDR double of kind $B$. In each case the rule number is copied from $A$ to $B .^{B}$

Several metarules can apply in the derivation of a single IDR double; bowever, the priaciple of Finile Closure, defined by Thompson (1982), allows every metarule to apply only once in the derivational history of each IDR double. The invocation of this principle avoids the derivation of infinite rule sets, in-

\footnotetext{
Blicle number might be a misleading lerm for $n$ because this copying assigns the same inceger to the whole class of rules that were derived from the same basic rules. This rule number propagation is a prerequisite for the CiPSG account of subcategorization.
}

cluding those that generate non-CF, non- $C S$, and nonrecursive languages. 7

Another component maps IDR doubles to IDR triples, which are ordered triples $(n, i, t)$ of a rule number $n$, an IDR $i$, and a translation t. The symbols of the resulting IDRs are fully instantiated feature sets (or structures) and therefore identical to object grammar symbols. Thus, this component adds semantic transiations and instantiates syntactic features. The mapping is controlled by a third set of rule extension principles including fealure co-occurrence restrictions, feature default principles, and an algorithm that assigns the right kind of translation to each rule on the basis of its syntactic information.

The last component of the metagrammar maps the IDR triples to the rules of the object grammar. For each IDR triple all the object grammar triples are generated whose CF-PS rules conform with the linear precedence (LP) rules, the fourth rule set of the metagrammar. LP rules are members of the LP relation, a partial ordering on $V_{T} \cup V_{N}$. An LP role $(\alpha, \beta)$ is usually written as $\alpha<\beta$ and simply states that $\alpha$ precedes $\beta$ whenever both $\alpha$ and $\beta$ occur in the right-hand-side of the same CF-PS rule

It is the separation of linear precedence from immediate dominance statements in the metagrammar that is referred to 2s ID/LP format. And it is precisely this aspect of the formalism that makes the theory attractive for application to languages with a bigh degree of word-order freedom. The analysis presented in the next section demonstrates the functioning of the formalism and some of its virtues.

\subsection{The Analysis of German Word Order}

l'szkoreit (1982a) proposes a GPSG analysis of German word order that accounts for the fixed-order phenomena, including the notoriously difficult problem of the position of finite and nonfuite verbs. Within the scope of this paper it is impossible to repeat the whole set of suggested rules. A tiny fragment should suffice to demonstrate the basic ideas as well as the need for unodifications of the framework.

Rule $(t)$ is the basic VP ID rule that combines ditransitive verbs like forms of geben (give) with its two objects:

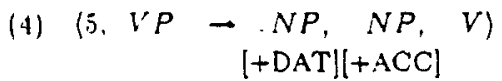

Thus rule stales that a VP can expand as a dative NP (IOBJ), an accusative NP (DOBJ), and a verb. Verbs that can occur in ditransitive VPs, like geben (give), are marked in the lexicon witb the rule number 5 . Nothing has been said about the linear order of these constituents. The following metarule supplies a "Gat" sentence rule for each main verb VP rule. [+NOM) stands for the nominative case, which marks the subject.

'Fi,r a discussion see Peters and Uszkoreit (1882) and Shieber et al. (1883). 
(5) $\underset{[-\mathrm{AUX}]}{V P}-X, V \Rightarrow S \rightarrow \underset{[+\mathrm{NOM}]}{N P, X, V}$

It generates the rule under (6) from (4):

(0) $\langle 5, S \rightarrow N P, \quad N P, \quad N P, \quad V\rangle$ $[+N O M][+D A T][+A C C]$

Example (7) gives a German constituent that will be admitted by a PS rule derived from ID rule ( $\theta)$ :

(7) der Doktor dem Mann die Pille gegeben the doctor the man the pill given

I shail not list the rules bere that combine the auxiliary hatte and the temporal adverb dann with (i) to arrive at sentence (1a), since these rules play no role in the ordering of the three noun phrases. What is of interest here is the mapping from ID rule ( 8$)$ to the appropriate set of PS rules. Which LP rules are needed to allow for all and only the acceptable linearizations?

The position of the verb is a relatively easy matter: if it is the finite matrix verb it precedes the noun phrases; in all other cascs, it follows everything else. We bave a feature MC for matrix clause as well as a feature co-occurrence restriction to casure that $+M C$ will always imply + FIN (finite). Two LP rules are needed for the main verb:

$(8 \mathrm{a})+\mathrm{MC}<\mathrm{NP}$

(8b)

$N P<-M C$

The regularities that govern the order of the noun phrases can also be encoded in LP rules, as in $(9 \mathrm{a})-(9 \mathrm{e})$ :

(0a) +NOMINATIVE $<$ +DATIVE
(9b) +NOMINATIVE $<$ +ACCUSATIVE
(0c) +DATIVE $<$ +ACCUSATIVE
(Od) -FOCUS $<$ +FOCUS
(0e) +PRONOUN $<$-PRONOUN

A feature FOCUS has been added that designates a focused constitucnt. Despite its a ame FOCUS is a syntactic feature, justified by syntactic racts, such as its influence on word order. This syntartic feature needs to be linked with the appropriate discourse information. The place to do this is in the rule extension component, where features are instantiated and semantic translations added to ID rules. It is assumed that in so doing the translation part of rules will have to be extended anyway so as to incorporate non-trutb-conditional aspects of the meaning. For example, the full translation could be an ordered pair of trutb-conditional and non-truth-conditional content, extending Karttunen and Peters's treatment of conventional implicature (hartunen and Peters, 1979) ${ }^{8}$ or a function from discourse situations to the appropriate truth-conditional meaning in the spirit of Barwise and Perry (1981). The analysis here is not concerned with cboosing a formalism for an extended semantic component, but ratber with demonstrating where the syntax has to provide for those elements of discourse information that influence the syntactic structure directly.

Note, that the new LP rules do not resolve the problem of ordering-principle conflicts, for the violation of one LP rule is euough to rule out an ordering. On the other hand, the absence of these LP rules would incorrectly predict that all permutations are acceptable. The next section introduces a redefinition of LP rules that provides a remedy for this deficiency.

\subsection{The Mlodified Framework}

Before introducing a new definition of LP rules, let me suggest another modification that will simplify things some hat. The L.P rules considered so far are not really LP rules in the sense in which they ware defined by their originators. After all. LP rules are defined as members of a partial ordering on $V_{N} \cup V_{T}$. Our rules are schemata for LP rules at best, abbreviating the buge set of LP rules that are instantiations of these scbemata. This definition is an unfortunate one in several respects. It not. only creates an unnecessarily large set of rules ( $V_{N}$ contains (housands of fully instantiated complex symbols) but also suppresses some of the important generalizations about the langunge. Clrarly, one could extract the relevant generalizations even from a fully expanded LP relation. e.g., realize that there is no LP rule whose first elemeat bas $-M C$ and its second element NP. However, it sbould not be necessary to extract generalizations from the grammar; the grammar should express these gencralizations directly. Another disadvantage follows from the choire of a procedure for arriving at the fully expanded LP relation. Stould all extensions that are compatible instantiations of $(8 \mathrm{a})$. (8b), and $(9 \mathrm{a})-(9 \mathrm{e})$ be LP rules? If so, then $(10)$ is an instantiation of $(8 \mathbf{a})$ :

$\begin{array}{ccc}(10)+M C & & . V P \\ +D E F & +F I N \\ & & \end{array}$

let nothing can be a matrix verb and definite simultaneously, and $\backsim P s$ cannot be finite. $(10)$ is a racuous rule. Whether it is a LP rulc at. all will depend on the way the nonterminal voc:b bulary of the object grammar is defined. If it ouly includes the conterminaly that actually occur in rules then $(10)$ is not a LP rule. In this case re would aced a component of the netagr ammar, the feature instantiation principles, to determine

\footnotetext{
${ }^{8}$ To be more precise, Karttunen and Peters actually make their transiatir ns urdered triples of truth-conditional content, implicatures, and an inheritance expression that plays a role in handling the projection problem for presuppositions.
} 
another component of the metagrammar, the LP component. ${ }^{9}$ $L P$ will be redefined as a partial order on $2^{F}$, where $F$ is the set of syntactic features 10

The second and more important change can best be described by viewing the LP component as a function from a pair of symbols (which can be characterized as feature sets) to trutb values, telling us for every pair of symbols whether the first can precede the second in a linearized rule. Given the LP relation $\left\{\left(\alpha_{1}, \beta_{1}\right\rangle,\left\{\alpha_{2}, \beta_{2}\right), \ldots,\left\langle\alpha_{n}, \beta_{n}\right\rangle\right\}$ and a pair of complex symbols $\langle\gamma, \delta\rangle$, the function can be expressed as in (11).

$$
\text { (11) } \begin{aligned}
& c_{1} \wedge c_{2} \wedge \ldots \wedge c_{n} \text { where } \\
& c_{i}=\neg\left(\alpha_{i} \subseteq \delta \wedge \beta_{i} \subseteq \gamma\right) \\
& \text { for } 1 \leq i \leq n
\end{aligned}
$$

We call the conjunct clauses LP conditions; the whole conjunction is a complex LP condition. The complex LP condition allows $\gamma$ to precede $\delta$ on the right-hand side of a $C F$. PS rule if every LP condition is true. An LP condition $c_{1}$ derived from the LP rule $\left\langle\alpha_{i}, \beta_{i}\right\rangle$ is true if it is not the ease that $\gamma$ bas the features $\beta_{i}$ and $\delta$ has the features $\alpha_{i}$. Thus the LP rule $N P<V P$ stands for the following nember of the LP relation $\{\{+N,-V,+2 B A R\},\{-N,+V,+2 B A R\}\}$. The LP condition following from this rule prevents a superset of $\{-N,+V,+2 B A R\}$ from preceding a superset of $\{-N .+V,+2 B A R\}$, i.e., a VP from preceding an $N P$.

But notice that there is nothing to prevent us from writing a fictitious LP rule such as

$$
\text { +PRONOUN }<\text {-ACCUSATIVE }
$$

Cierman bas verbs like lehren that take two accusative noun phrases as complements. If (12) were an LP rule then the resulting LP condition defined as in (11) would rule out any oceurrence of (wo pronominalized siscer NPs because either order would be rejected. ${ }^{11}$

It. is an empirical question if one might ever find it useful to write L.P rules as in (12), i.e., rules $a<\beta$, where $a \cup \beta$ could be a cubset of a complex symbol. Let me introduce a minor redefinition of the interpretation of LP, which will take carc of cases such as (12) and at the same prepare the way for a more substantial modification of LP rules. LP shall again be interpreced as a function from pairs of feature sets (associated with complex symbols) to trutb values. Given the LP relation $\left\{\left(\alpha_{1}, \beta_{1}\right\rangle,\left(\alpha_{2}, \beta_{2}\right), \ldots,\left(\alpha_{n}, \beta_{n}\right)\right\}$ and a pair of complex symbols

\footnotetext{
OThe widely used notation for noninstantiated LP rules and the festure in stantiatirin principles could be regarded as meta-melagrammatical devices that inductively define a part of the metagrammar.

10 Remember that, in an $\bar{X}$-syntax, syntactic calegories abbreviate feature sets $N P=\{+N,-V,+2 B A R\}$. The definition can evoily be extended to work on feature trees instead of feature sets.

11 In principle, there is nothing in the original $\mathrm{DD} / \mathrm{LP}$ definition either that would prevent the grammar writer from abbreviating a set of LP rules by (12). It is not quite clear, however, which set of LP rules is abbreviated by $(12)$.
}

$(\gamma, \delta)$, the function can be expressed as in (13).

$$
\text { (13) } \begin{aligned}
& c_{1} \wedge c_{2} \wedge \ldots \wedge c_{n} \text { where } \\
& c_{i}=\left(\alpha_{i} \subseteq \delta \wedge \beta_{i} \subseteq \gamma\right) \rightarrow\left(\alpha_{i} \subseteq \gamma \wedge \beta_{i} \subseteq \delta\right) \\
& \text { for } 1 \leq i \leq n
\end{aligned}
$$

That means $y$ can precede $\delta$ if all LP conditions are true. For instance, the LP condition of LP rule (12) will yield false only if $\gamma$ is +ACCUSATIVE and $\delta$ is +PRONOUN, and either $\gamma$ is -PRONOUN or $\delta$ is -ACCUSATIVE (or both).

- Now let us assume that, in addition to the kind of simple LP rules just introduced, we can also bave complex LP rules consisting of several simple LP rules and notated in curled brackets as in (14):

$$
\text { (14) }\left\{\begin{array}{ll}
+ \text { NOMINATIVE } & <\text { +DATIVE } \\
+ \text { NOMINATIVE } & <\text { +ACCUSATIVE } \\
+ \text { DATIVE } & <\text { +ACCUSATIVE } \\
\text {-FOCUS } & <\text { +FOCUS } \\
+ \text { PRONOUN } & <\text {-PRONOUN }
\end{array}\right\}
$$

The LP condition associated with such a complex LP rule shall be the disjunction of the LP conditions assigned to its members. LP rules can be generally defined as sets of ordered pairs of feature sets $\left\{\left(\alpha_{1}, \beta_{1}\right\rangle,\left\{\alpha_{2}, \beta_{2}\right\rangle, \ldots,\left\langle\alpha_{m}, \beta_{m}\right\rangle\right\}$, which are either notated with curled brackets as in $(10)$, or, in the case of singletons, as LP rules of the familiar kind. A complex LP rule $\left\{\left(\alpha_{1}, \beta_{1}\right\rangle,\left\langle\alpha_{2}, \beta_{2}\right\rangle, \ldots,\left\langle\alpha_{m}, \beta_{m}\right\rangle\right\}$ is interpreted as a LP condition of the following form $\left(\left(\alpha_{1} \subseteq \delta \wedge \beta_{1} \subseteq \gamma\right) \vee\left(\alpha_{2} \subseteq \delta \wedge \beta_{2} \subseteq\right.\right.$ भ) $\left.\vee \ldots \vee\left(\alpha_{m} \subseteq \delta \wedge \beta_{m} \subseteq \gamma\right)\right) \rightarrow\left(\left(\alpha_{1} \subseteq \gamma \wedge \beta_{1} \subseteq \delta\right) \vee\left(\alpha_{2} \subseteq\right.\right.$ $\left.\left.i \wedge \beta_{2} \subseteq \delta\right) \vee \ldots \vee\left(\alpha_{m} \subseteq \gamma \wedge \beta_{m} \subseteq \delta\right)\right)$. Any of the atomic LP rules within the complex LP rule can be violated as long as the violations are sactioned by at least one of the atomic LP rules.

Notice that with respect to this definition. "regular" LP rulcs, i.e., singletons, can be regarded as a special case of complex L.P rules.

I want 10 suggest that the LP rules in $(8 a),(8 b)$, and $(1-4)$ are a subset of the LP rules of German. This analysis makes a number of empirical predictions. For example, it predicts that (15) and (10) are grammatical, but not (17).

(15) Dann batte der Doktor dem Mann die Pille gegeben $\begin{array}{ll}- \text { FOCUS } & + \text { FOCUS } \\ + \text { NOM FOCUS } & \text { +DAT }\end{array}$

Then had the doctor the man the pill given

(16) Dann hatte der Doktor die Pille dem Mann gegeben -FOCUS +FOCUS +FOCLS $+\mathrm{NOM}+\mathrm{ACC}+\mathrm{DAT}$

Then had the doctor the pill the man given

(17)??Dann hatte der Doktor die Pille dem Mann gegeben -FOCUS +FOCUS -FOCUS + NOM +ACC +DAT

Then had the doctor the pill the man given 
In (17) the sub-LP-rules +DAT < +ACC and -FOCUS < + FOCUS are violated. No other sub-LP-rule legitimizes these violations and therefore the sentence is bad.

This agrees with the findings of Lenerz (1977), who tested a large number of sample sentences in order to determine the interaction of the unmarked syntactic order and the ordering preferences introduced by discourse roles. There are too many possible feature instantiations and permutations of the three boun phrases to permit making grammaticality predictions here for a larger sample of ordering variants. So far I bave not discovered any empirical deficiencies in the proposed analysis.

\section{Implications for Implementations}

The theory of GPSG, as described by its creators and as outlined in this paper, cannot be used directly for implementation. The number of rules generated by the metagrammar is just too large. The Hewlett-Packard system (Gawron et al., 1982) as well as Henry Thompson's program, which are both based on a pre-ID/LP version of GPSG, use metarules as metagrammatical devices. but with feature instantiation built into the processor. Agreement checks, however, which correspond to the work of the metagrammatical feature instantiation principles, are done at parse time. As Berwick and Weinberg (1982) have pointed out, the context-ireeness of a grammar might not accomplist much when the number of sules explodes. The more components of the metagrammar that can be built into the processor (or uscd by it as additional rule sets at parse time), the smaller the resulting grammar will be. The task is to search for parsing algorithms that incorporate the work of the metagrammar iato context-free phrase structure parsing without completely losing the parsing time advantages of the latter. Most PSG parsers do feat ure bandling at parse time. Recently, Shieber (forthcoming) bas extended the Earley algorithm (Earley 1970) to incorporate the linearization process without a concomitant loss in parsing efficiency. The redcfinition of the LP component proposed in this paper can be integrated easily and efficiently into Shieber's extension.

If the parser uses the disjunctive LP rules to accept all ordering variants that are well-formed with respect to a discourse, there still remains the question of how the generator chooses among the disjuncts in the LP rule. It would be very surprising if the different orderings that can be obtained by choosing one LP rule disjnact over another did in fact occur with equal frequency. Alt hough there are no clear results that might provide an answer to this question, there are indications that certain disjuncts "win out" more often than others. However, this choice is purcly stylistic. A system that is supposed to produce bighquality output might contain a stylistic selection mechanism that avoids reprlitions or chooses among variants according to the type of text or dialogue.

\section{Conclusion}

The proposed analygis of partially free word order in German makes the accurate predictions about the gram- maticality of ordering variants, including their appropriateness with respect to a given discourse. The ID/LP lormat, which has the mechanisms to handle free word order, has been extended to account for the interaction of syntax and pragmatics, as well as for the mutually competing ordering principles. The modifications are compatible with efficient implementation models. The redefined LP component can be used for the implcmentation of stylistic choice.

\section{References}

Barwise, J. and J. Perry (1981) "Situations and Attitudes", Journal of Pbllosophy, 1981, 668-691.

Berwick, R. C., and A. S. Weinberg "Parsing Efliciency, Computational Complexity, and the Evaluation of Grammatical Theories," Lloguiotle Inquiry, 13, 165-191.

Earley, J. (1970) "An Efficient Context-Free Parsing Algorithm," Commualeatlone of the ACM, 13, (1970), 94-102.

Gawron. M. J.. et al. (1082) "The GPSG Linguistics System." Proceedinge of the 20th Aanual Menting of the Aseociation for Computational Liaguintica. University of Toronto, Toronto, June 1982, 74-81.

Gazdar. G. and G. Pullum (1981) "Subcategorization, Constituent Order and the Notion 'llead"," in M. Moortgat, H.v.d. Hulst and T. Hoekstra. eds., The Seope of Lexical Rules. 107. 1:3. Foris, Dordrecht, Holland, 1081.

Gazdar. G. and G. Pullum (1082) "Generalized Phrase Structure Grammar: A Theoretical Synopsis." Indiana University Linguistics Club, Bloomington, Indiana.

Gazılar. G., (i. Pullum, and I. Sag (1981) "Auxiliaries and related phenomena in a restrictive theory of grammar, "Laguage 58, $591-638$.

Kamp, H. (1980) "A theory of truth and semantic representation" ms.

Kiartunen, L. and S. Peters $(1079)$ "Conventional implicature," in C. K. Oh and D. Dinneen (eds.), Syatax ad Somanties, Vol. 11: Presupposition, Academic Press, New York, 1-56.

Kinin. E. (108.?) A Mluitiset Analysis of Immediate Domination Rules" ins.

Lenerz. J. (10Ti) Zur Abfolge aomianlez Satuglieder Im Deutachen, TBL Verlag Gunter Narr. Tuebingen, 1077.

Montagur, R. (1074) Formal Phllosophy, edited and with an intro durtion lyy R. Thomason, Yale Lniversity Press, New Haven.

Nerhornc. J. (108:2) "Phantoms' in German fronting: Poltergeist constituents? - paper presented at the 1982. Annual meeting of the Linguistic Society of America. San Diego. California, December 108?.

Peters, S. and 1I. "szkoreit, "Essential Variables in Metarules," paper presented at the 1082 Annual Meeting of the Linguistic Society of America, San Diego, California, December 1982.

Pullum, G. (108:) "Free Word Order and Phrase Structure Ruirs." J. Pustejorsky and P. Sells, (eds.), Proceeding* of the Treltth Annual Meetlog of the North Eertern Lloguletin Soelety, Graduate Linguistics Student Association, University of Massachusetts, Amherst, Massachusetts 1982.

Shicher, S. (forthcoming) "Direct Parsing of D/LP Grammars."

Uszkoreit. H. (1982a) "German Word Order in GPSG." in D. Flickinger, M. Macken, and N. Wiegand (eds.). Proesodings 
of the Firat Went Cont Conference on Formal

Lincuiatles, Stanford University, Stanford, California (1982).

('szkoreit, H. (1982b) "Topicalization in Standard German," paper presented at the 1982 Annual meeting of the Linguistic Society of America, San Diego, December 1982. 